\title{
Correction to: Timing of thoracic radiotherapy is more important than dose intensification in patients with limited-stage small cell lung cancer: a parallel comparison of two prospective studies
}

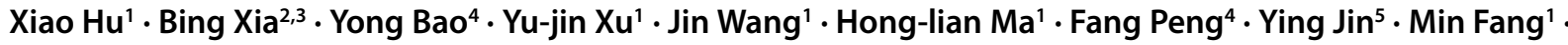 \\ Hua-rong Tang ${ }^{1} \cdot$ Meng-yuan Chen ${ }^{1} \cdot$ Bai-qiang Dong ${ }^{1} \cdot$ Jia-nan Jin ${ }^{1} \cdot$ Xiao-long Fu ${ }^{3,6} \cdot$ Ming Chen ${ }^{1}$
}

Published online: 20 February 2020

(c) Springer-Verlag GmbH Germany, part of Springer Nature 2020

\section{Correction to:}

\section{Strahlenther Onkol 2019}

https://doi.org/10.1007/s00066-019-01539-1

The original version of this article unfortunately contained a mistake. The correct version of the funding information are given below.

Funding This study was funded by the National Natural Science Foundation of China (grant numbers 81402540 and 81672972), the National Health Commission scientific research funds-Zhejiang province major science and technology project on medicine (grant number WKJ-ZJ-1701), co-cultivation project of National Health Commission of the People's Republic of China and Zhejiang province (grant number 2014PYA003), and Zhejiang Science and Technology Plan on Medicine and Health (grant number 2019KY046) and Western Medicine Guidance Project of Shanghai Science and Technology Commission (Grant No. 16411968200).

The online version of the original article can be found under https://doi.org/10.1007/s00066-019-01539-1.

Xiao-long $\mathrm{Fu}$

xlfu1964@126.com

$\triangle$ Ming Chen

chenming@zjcc.org.cn

1 Department of Radiation Oncology, Cancer Hospital of the University of Chinese Academy of Sciences, Department of Radiation Oncology, Zhejiang Cancer Hospital, Zhejiang Provincial Key Laboratory of Radiation Oncology, Institute of Cancer Research and Basic Medical Sciences, Chinese Academy of Sciences, 1 Banshan Road East, 310022 Hangzhou, China
2 Department of Radiation Oncology, Hangzhou Cancer Hospital, Hangzhou, China

3 Department of Radiation Oncology, Cancer Hospital of Fudan University, Shanghai, China

4 Department of Radiation Oncology, The First affiliated Hospital of Sun Yat-sen University, Guangzhou, China

5 Department of Medical Oncology, Cancer Hospital of the University of Chinese Academy of Sciences, Department of Medical Oncology, Zhejiang Cancer Hospital, Institute of Cancer Research and Basic Medical Sciences, Chinese Academy of Sciences, Hangzhou, China

6 Department of Radiation Oncology, Shanghai Jiao Tong University Affiliated Chest Hospital, 241 Huaihai Road West, 200030 Shanghai, China 